\title{
Immunopathological Studies on Diseases of the Liver
}

\author{
Hideo NAGASHIMA \\ The First Department of Internal Medicine \\ Okayama University Medical School
}

\section{Introduction}

In this study, an attempt was made to investigate how allergic mechanism plays an important role in inflammatory liver diseases, especially in virus hepatitis, and also whether the auto-immune process causes protracting pathologic change to the liver or not except for primary pathogeny.

\section{Materials and Methods}

1. Preparation of antigen:

$5 \%$ homogeneous (human being, rabbit, mouse) or heterogeneous (guinea pig) liver extract with physiological saline solution. 5\% Ovalbumin solution.

2. Technique of antibody estimation:

Complement fixation reaction, precipitation reaction, and immunodiffusion techniques [single diffusion (Oudin), double diffusion (Ouchterlony)], immunoelectrophoresis were used.

Liver cell (or lymph cell, prepared from mesenteric lymph glands, erythrocyte, prepared from pheripheral blood) suspensions obtained from the patients suffering from liver diseases by biopsy, and from rabbits, guinea pigs and mice were prepared with Tyrode solution at $37^{\circ} \mathrm{C}$, which were slightly homogenized, and then washed several times with $9 \%$ sucrose solution (ph 7.2, ionic strength 0.016). The anamnestic reaction could be induced by the administration of ACE (adrenocortical extract) (4 ml./day for 2 days).

For cell electrophoresis, the liver cells (or lymph cells, erythrocytes) were suspended in $9.0 \%$ sucrose solution, and the cell electrophoretic mobilities were measured by the electrophoretic apparatus 


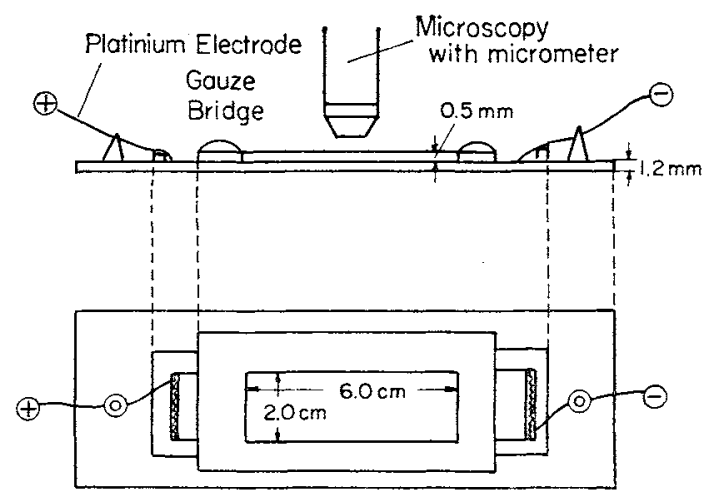

Fig. 1. Electrophoretic apparatus.

Electrophoretic condition: electric pressure 300 volt electric current $0.3 \sim 0.5 \mathrm{~mA}$. distance $6.0 \mathrm{~cm}$. cell medium 9.0\% sucrose solution electrophoretic mobility $\mu / \mathrm{sec}$.

shown in Fig. 1.

In the immunocytological experiments, the liver cells in Tyrode solution ( $4 \mathrm{ml}$.) with the corresponded antigen (the sensitized antigen). or antiserum $\left(0.4 \mathrm{ml}\right.$.) were incubated at $37^{\circ} \mathrm{C}$. for $1-3$ hours.

The succinic dehydrogenase activity was estimated by Ichikawa's method. For the morphological examinations of the liver tissues, electron microscopic observation, hematoxylineosin stain method etc. were employed.

\section{Sensitization}

-In view of host-parasite relationship-

The correlations between the changes in body fluid and the morphological change were studied on the experimental ectromelia virus hepatitis in mice. The antibody against ectromelia virus and the direct Coombs' antibody were produced about 4 to 10 days after the intraperitoneal inoculation of ectromelia virus, but the appearance of the auto-liver antibody was seen about 20 days later. The activated function in the reticuloendothelial system was confirmed by the high succinic dehydrogenase activity in the circulating leukocytes, the liver and spleen tissues. The elementary bodies of ectro- 
melia virus were observed in the liver tissue 2 to 5 days after inoculation of the virus, and the demarcation of the necrotic area. became shaxp about 12 days later. And the positive direct Coombs' test at the early stage of ectromelia virus infection may indicate that the ectromelia virus is attached to the surface of erythrocytes, since electrophoretic mobilities of erythrocyte became normal by isolating the virus at $37^{\circ} \mathrm{C}$ from the erythrocyte of inoculated mice. It was shown that the mouse sensitized with homologous liver emulsion and ovalbumin was less resistant to the viral infection. compared with non-sensitized one, and the histological study revealed that colliquative necrosis, separation of the hepatic cord, exudation and cell infiltration in the lobules and the Glisson's capsule were more extensive than those in the non-sensitized one, thus the serous. inflammation was more characteristic in the sensitized one.

\section{Discussion on the auto-sensitization and the auto-antibody producing mechanism}

The production of the anti-homologous liver antibody by inoculating the rabbit with the homologous liver emulsion and moreover the auto-antibody production by injecting $10 \%$ aseptic $\mathrm{NaCl}$ solution into the liver of the rabbit were successful.

A tendency of decrease in total serum protein and serum albumin and of increase in $r$-globulin was observed in the sensitization processes by the liver emulsions, but the elevated level of histamine in the blood was not observed. The homologous liver antibody production was detected by the agar precipitation reaction by the Oudin's method. The anti-homologous liver antibody in the liver, spleen and kidney etc. were found by the complement fixation reaction, but the homologous liver antibody in those organs was very low in titer compared with that in the peripheral blood. The anamnestic reaction by the intravenous administration of the corresponding antigen or ACE was able to be induced in the stage of disappearance of the homologous-liver antibody and autoliver-antibody.

At the anamnestic reaction, the corresponding antibody titer was elevated in parallel to the antibody titer of the sensitizing stage. And at the cross reaction of the antibody with the tissue antigens 
extracted from various abdominal organs, the antibody induced at the anamnestic reaction had relative affinity to the corresponding tissue antigen. The blood picture at that time revealed that the lymphocyte decreased in number. The anamnestic reaction could not be induced by the premanagement with nitromin injection. The histological changes by sensitization were active reaction of the Kupffer's cells, the appearance of pyroninophile Kupffer's cell, a slight cell infiltration into the Glisson's capsule, but the degeneration and necrosis of liver cells were scarcely observed, the hyperfunction of the liver cell, the hypertrophy of the lymph follicle, hyperplastic reaction with the plasma cell proliferation in the vicinity of the medullary arteries, antigen-phagocytic and hyperphagocytic reactions of sinus endothelium, reticulocytes and free histiocytes were also observed in the spleen. Similar morphological changes were observed in cases of anti-liver damage by injecting 10\% aseptic $\mathrm{NaCl}$ solution into the liver of the rabbit. The fraction of the liver tissue by ultracentrifuge was separated in 4 components, and each fraction was used as the antigen in complement fixation reaction, and the microsome fraction reacted a little more remarkably to the auto-liver antibody than the homologous liver antibody.

The rabbits inoculated with the rabbits liver microsome fraction produced the homologous liver antibody. At that time morphological changes were similar to those of the rabbits sensitized with the above described antigens. The electron microscopic findings of the liver cell sensitized with the auto- and homologous liver emulsion indicated the swelling and disarrangement of the endoplasmic reticulum, decrease of RNA granules, normal mitochondria, the change of cell granulation structure in cytoplasmic ground substance.

The electrophoretic mobility of the sensitized liver cells with the above described antigen remarkably increased than that of normal rabbit liver cells, and at the decreased stage in anti-liver antibody titer the electrophoretic mobilities decreased in parallel with the anti-liver antibody titer, and in the anamnestic reaction the electrophoretic mobilities increased. Moreover, after the incubation for 1 to 3 hours in Tyrode solution with $1 / 10$ dose of the corresponding antigen or antibody at $37^{\circ} \mathrm{C}$, the swelling of the central region of the protoplasm, roughness of the mitochondria, variation 
in size of storage granules, change of the staining of cytoplasmic granular substances and blister formation were observed in the sensitized liver cell or in the liver cell of anamnestic response by means of phase contrast microscopy. The same changes were seen in the lymphocytes in mesenteric lymph glands in a similar manner. These immunocytological changes became normal reversively as the sensitization disappeared. It was proved by these results that the above described phenomena were based on the change of general sensitizing state, as the same findings were observed in the sensitized rabbits with heterologous protein (ovalbumin) (Fig. 2). The electrophoretic mobilities of the liver cell of the positive auto-liver antibody cases were increased by addition of the serum containing homologous human liver antibody, compared with the negative autoliver antibody cases.

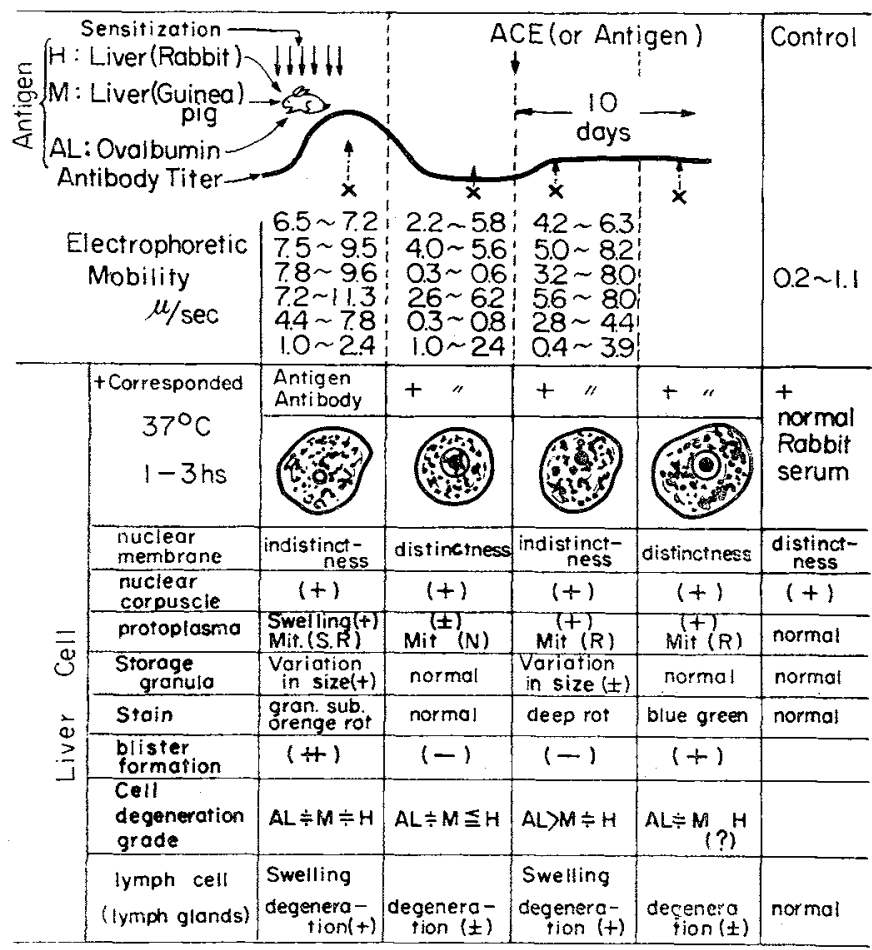

Fig. 2. The reactivity of the liver cell and lymph cell in sensitization.

Mit: Mitochondria S: Swelling R: Roughness gran. sub. : granular substances 
In the cases of virus hepatitis the electrophoretic mobilities of the liver cell in the active stage increased a little more than that of compensatory stage, and in chronic virus hepatitis cases, the electrophoretic mobilities of the liver cell increased a little more than that of acute virus hepatitis cases.

In vitro, when the anti-liver antibody serum was added to the liver cells of patients, who had positive auto-liver antibody in the Tyrode solution, the liver cells manifested indistinctness of nuclear membrane, disappearance of nuclear corpuscle, more promoted roughness of granular substance in the protoplasm, and more increased electrophoretic mobilities than the negative auto-liver antibody cases of virus hepatitis.

In the positive anamnestic reaction cases of hepatitis and liver cirrhosis, there were many cases which showed degeneration of the liver cell, cell infiltration, proliferation of the interstitium, the activation of Kupffer's cell, and increased exudation in the sinus endothelium.

It was considered from the above-stated results that these phenomena were based on the change of the sensitized general state to the sensitization and did not depend upon the abnormality of the antibody formation system.

At the early stage of virus hepatitis the appearance of autoantibody was observed, and the rapid recovery of clinical pictures during the course of the auto-immune phenomenon in the cases of immunohemolytic anemia in virus hepatitis supported the above idea.

\section{Antigen-antibody reaction system and liver}

-Especially on hepatitis and its protracting process-

The appearance of the auto-liver antibody increased in percentage and an elevated titer of auto-antibody was obtained at the chronic stage of virus hepatitis. The direct Coombs' test showed a high positive rate at the early stage of the illness, and the complement decreased in titer at the acute early stage and chronic recurrent stage of virus hepatitis. The change of properdin level was not remarkable at the acute early stage and the positive percentage of C-reactive protein was high at the acute early stage $(75 \%)$ of virus 
hepatitis. It was noticed that many cases of the positive auto-liver antibody cases were clinically the protracting cases $(33.3-53 \%)$ and the compensatory cases $(16.7-50 \%)$ on the course of virus hepatitis more than 3 months after onset of the illness, on the other hand, 33.2-83.3 per cent of the negative auto-liver antibody cases were the improved cases.

The percentage of the positive direct Coombs' test was about the same in each stage of virus hepatitis, ranging from 25 to $27.8 \%$ and was not necessarily high among chronic cases with hemolysis.

An elevated level of the serum transaminase activity of $42.1 \%$ was followed by the appearance of the auto-liver antibody in peripheral blood.

It was conceivable that there existed a relatively close correlation between the production of the auto-liver antibody and the protracting process of hepatitis, and from the clinical observation the pathogenic significance of the auto-liver antibody could not be denied. Moreover, it was confirmed that the qualitative change of the antibody formation system did not play an important role in the appearance of the auto-antibodies in view of the clinical findings and also the positive direct Coombs' test cases were not usually accompanied by hemolytic phenomena, whose positive rate was proved to be not increased even in cases of protracting process of virus hepatitis.

\section{Studies on the auto-immune phenomenon in hepatitis and liver cirrhosis}

\section{-Clinical studies on the auto-sensitization-}

The clinical pictures in the course of virus hepatitis were studied from the immuno-histological point of view, especially the correlations between the changes of body fluid and morphological findings were observed.

Group I consisted of 22 cases with negative auto-liver complement-fixation reaction and negative direct Coombs' test; group II, 10 cases associated with only positive Coombs' test; group III, 21 cases associated with only positive auto-liver complement-fixation reaction and group IV, 12 cases in which the auto-liver complement- 
fixation reaction as well as the direct Coombs' test were positive (Fig. 3).

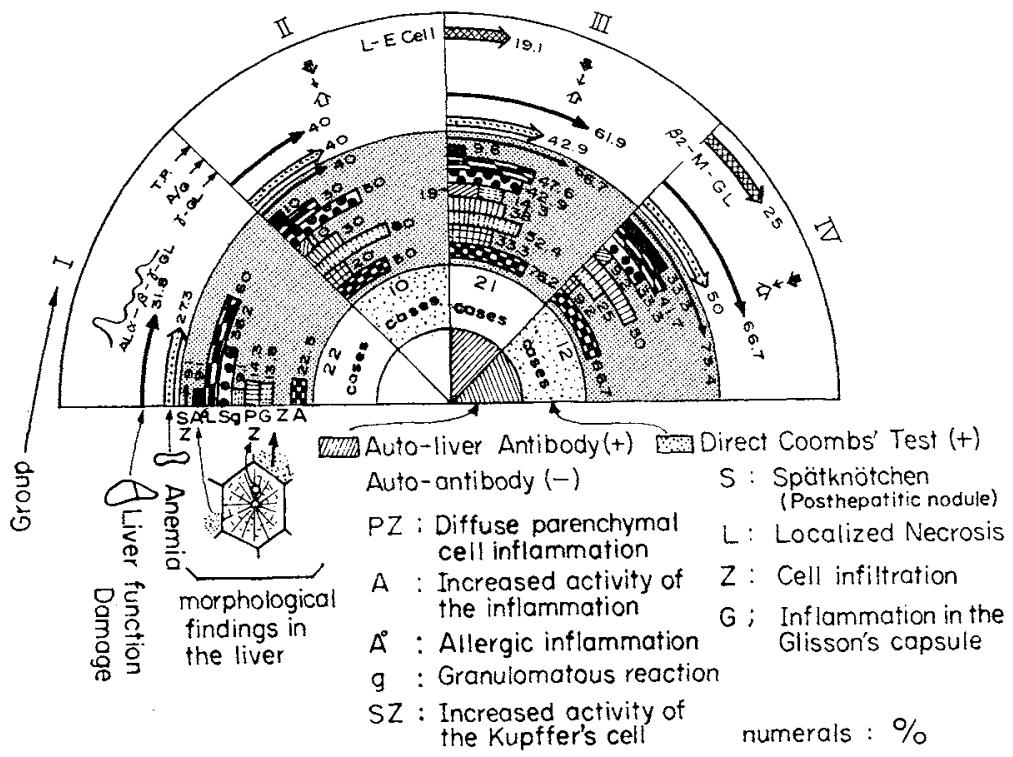

Fig. 3. Clinical studies on the auto-sensitization.

As far as morphological changes were concerned, the intensity of inflammation became stronger in the following order : I<II $<$ III $<I V$. And the diffuse inflammatory changes in the liver parenchyma were extensive in the same order. The allergic reaction caused by angitis and its perivascular reticulation was remarkable in the group IV. The granulomatous reaction was frequent in the group III and, on the contrary, the localizing tendency of the inflammation in the Glisson's capsule, Buchner's posthepatitic nodule (Spätknötchen) and localized necrosis showed an increasing tendency in the following order : IV $<$ III $<$ II $<$ I.

As to the correlation between the immunological reactions and histological findings, 54.6\% of the cases of the group I showed the inflammation of the liver parenchyma, the incidences of the inflammation of the Glisson's capsule and the inflammation of the liver parenchyma were $40 \%$ and $30 \%$ respectively in the group II, the precirrhosis of the liver and the protracting process to liver cirrhosis were found in $4.8 \%$ and $52.7 \%$ respectively in the group III and 
many cases of liver cirrhosis were also found in the group IV. The cases of anemia became to increase in the following order: $\mathrm{I}<\mathrm{II}<$ III $<$ IV. And the cases with liver function damage also increased in number in the same order. There was a slight decrease in total serum protein concentration, and the decline of the albumin-globulin ratio and the high $r$-globulin concentration were observed in the serum protein picture, and the appearance of $\beta_{2-M}$-globulin and of the $\mathrm{L}-\mathrm{E}$ cell or $\mathrm{L}-\mathrm{E}$ phenomenon was seen in $19.1 \%$ of the group III and $25.0 \%$ of the group IV.

The hemolytic phenomena were immunohematologically caused in some cases of virus hepatitis with the positive direct Coombs' test, while the increase of the electrophoretic mobility of the liver cell was seen in many of the positive auto-liver antibody cases and the decrease of the electrophoretic mobility of erythrocyte was seen in the group III in spite of the negative direct Coombs' test, but at the same time the elevated serum plasmin activity was not observed. It was, therefore, pointed out that there might be various processes to develop the hemolytic anemia.

And the activity of the reticuloendothelial system increased gradually in the following order: I $<$ II $<$ III $<$ IV.

The decrease in the auto-liver antibody titer was observed in 10 of the 15 virus hepatitis cases on the administration of the steroid hormones (prednisolone, dexamethazone) to the positive autoliver antibody cases. And the liver function, the serum albumin picture and the histological appearance etc. became normal at that time, but the appearance of the auto-liver antibody was seen by administration of steroid hormones in the peripheral blood in few cases of negative auto-liver antibody, in which the histological and functional changes of the liver were observed.

Summarizing the above results, the diffuse inflammation, the increase of inflammatory activity of the liver, the protracting process to liver cirrhosis and the appearance of allergic reaction were morphologically observed in the cases with the positive auto-antibody, and also the change of the serum protein picture, the disturbance of the liver function and the increase in anemia were observed at the same time as changes of the body fluid and those changes were clearly understood to be due to the increase of the sensitization. 
And it was thought that the above described animal experiments could explain these clinical phenomena. The negative auto-antibody reaction meant the decline in the grade of sensitization and the localizing tendency of the necrosis and the normality of the body fluid lent support to the above fact. And it was shown that the allergic mechanism in the inflammation of the liver should be understood as a partial phenomenon of the general sensitizing state. Though it was important for the problem of the localizing antibody, it was very difficult to find out the specificity to the liver parenchyma morphologically in the studies of the localization of antibody in various organs after injection of the atoxyl-labelled rabbits' anti-liver serum into rabbits, but the distribution of the atoxyllabelled homologous liver antibody in the liver was relatively more in comparison with the control animal which was injected with atoxyl-labelled normal rabbits serum.

A remarkable decrease in the serum albumin concentration and the liver damage were clearly observed in the cases with the administration of the anti-liver serum (homologous and heterologous liver antiserum) in comparison with the control animal with the administration of the heterologous stomach and intestine antiserum.

Those facts suggested that the relative affinity of the antihomologous liver serum to liver parenchyma and the liver damage were induced.

In the non-sensitized rabbits, the electrophoretic mobility of the liver cell or lymph cell decreased by addition of the anti-liver serum to the liver cell or lymph cell of non-sensitized rabbits in Tyrode solution, but remarkable changes were not observed by phase contrast microscopy. On the contrary, the electrophoretic mobility of the liver cell became increased by addition of the anti-liver serum in the sensitized liver cell, and the remarkable changes as above described were observed by means of phase contrast microscopy of the liver cell. A rapid intracellular metabolic change was recognized from the change of electric change density of the liver cell by incubating the liver cell with an auto-liver antibody serum, the morphologic changes of granular substances, the blister formation and the change of staining character etc. in the liver cells were caused on the sensitization, and these similar findings above men- 
tioned were seen in the cases with the addition of the liver antigen. In view of these phenomena it seemed to suggest that there could be a cell fixed antibody. And the decreased electrophoretic mobility of the liver cells in ectromelia virus infected mice and erythrocytes could also be observed.

Therefore, it may be concluded that the reactivity of the liver cell and lymph cell is quite different between the stage of hypersensitiveness and the non-sensitization stage, or the decreased stage of hypersensitiveness.

\section{Conclusion}

In view of the examination of the body fluid and morphological appearances in the inflammatory process of the liver, the clinical pictures of the auto-sensitization have been demonstrated. It is felt that the changes of the reactivity of the liver cells are based on a partial phenomenon of the general sensitizing state and also the proper function of the liver should be considered to play an important role in development of allergic mechanism of the liver.

\section{References}

1) Burnet, F.M.: The Integrity of the Body: A discussion of modern immunological idea, Harvard. U.P. 1962.

2) Medawar, P. B.: Kagaku $31: 244,1961$.

3) Nagashima, H.: J. Jap. Assoc. Infect. Dis. $55: 574,1958$.

4) Nagashima, H.: Nissin Igaku $48: 571,1961$.

5) Vorlaender, K. O.: Z. ges. exp. Med. 118: 352, 1952.

6) Miescher, P. A.: Dtsch. Med. Wschr. 85:706, 1960.

7) Hogeboom, G.H., Schneider, W. C. \& Palade, C. E.: J. Biol. Chem. 172: 619, 1948.

8) Daemshek, K.: Acta Haem. Jap. 23: No. 6, Supp1. 83, 1960.

9) Meyer-Krahmer, H. G.: Z. ges. exp. Med. 116:390, 1950.

10) Pillemer, L. et al.: Sci. 121:732, 1955.

11) Olotzki, L. et al: J. Infect. Dis. 77:66, 1945.

12) Muto, K.: Arch. exp. Path. Pharmak. 621: 494, 1909.

13) Ichikawa, O.: Kagaku 25: 89, 1958.

14) Ishii, K.: Jap. J. Gastroent. 54: 1, 1957.

15) Ishii, K.: Acta Hepatologia Jap. 2: 400, 1961.

16) McIntire, F.C. et al.: J. Biol. Chem. 170:537, 1947.

17) Suzuki, K.: Jap. J. Allergy 8: 541, 1959.

18) Kosaka, K. and Nagashima, H.: Nippon Rinsho $20: 25,1962$. 